\title{
Relação dos pareceristas ad hoc do v. 9, n. 1-2, 2018
}

\section{Editora}

Maria Inês Côrte Vitoria PUCRS, RS, Brasil

\section{Editora Colaboradora}

Pricila Kohls dos Santos PUCRS, RS, Brasil

\section{Equipe Editorial}

Carla Spagnolo PUCRS, Brasil

Rosa Maria Rigo

PUCRS, Brasil

\section{ISSN 2179-8435}

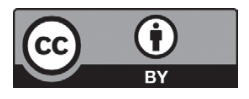

Este artigo está licenciado sob forma de uma licença Creative Commons Atribuição 4.0 Internacional, qu qualquer meio, desde que a publicação original seja corretamente citada. gradecemos a todos os pareceristas abaixo, que colaboraram com a revista Educação Por Escrito no ano de 2018:

- Altair Alberto Fávero (Universidade de Passo Fundo)

- Beatriz Diconca Hernandez (Universidad de la Republica - Uruguay)

- Carla Spagnolo (Pontifícia Universidade Católica do Rio Grande do Sul)

- Dirce Hechler Herbertz (Pontifícia Universidade Católica do Rio Grande do Sul)

- Eliana Ortiz Castilla (Universidad del Rosário - Colômbia)

- Glaé Corrêa Machado (Centro Universitário La Salle)

- Jordana Wruck Timm (Universidade Regional Integrada do Alto Uruguai e das Missões)

- Lorena do Nascimento (Pontifícia Universidade Católica do Rio Grande do Sul)

- Marilene Batista da Cruz Nascimento (Universidade Federal de Sergipe)

- Marilene Gabriel Dalla Corte (Universidade Federal de Santa Maria)

- Martha Luci Sozo (Universidade Federal de Ciências da Saúde de Porto Alegre)

- Nozangela Maria Rolim Dantas (Universidade Federal de Campina Grande)

- Patricia Estrada Mejia (Universidad de Antioquia - Colômbia)

- Pricila Kohls dos Santos (Pontifícia Universidade Católica do Rio Grande do Sul)

- Rosa Maria Rigo (Pontifícia Universidade Católica do Rio Grande do Sul)

- Rosália Figueiró Borges (Universidade do Vale do Rio dos Sinos)

- Raimunda Cunha Ribeiro (Universidade Estadual do Piauí)

- Rodrigo Avila Colla (Pontifícia Universidade Católica do Rio Grande do Sul)

- Tatiane de Freitas Ermel (Universidade Regional Integrada do Alto Uruguai e das Missões)

- Viviane Guidotti (Pontifícia Universidade Católica do Rio Grande do Sul)

- Zoraia Aguiar Bittencourt (Universidade Federal da Fronteira Sul) 